Review Article

\title{
Efficacy and Safety of Aidi Injection as an Adjuvant Therapy on Advanced Breast Cancer: A Systematic Review and Meta-Analysis of Randomized Controlled Trials
}

\author{
Yihui Chai, ${ }^{1}$ Yunzhi Chen, ${ }^{1,2}$ Wen Li $\left(\mathbb{D},{ }^{1,3}\right.$ Zhong Qin, ${ }^{1}$ Jie Gao, ${ }^{1}$ Zhibin Jiang, \\ Yuhong Ge, ${ }^{1}$ Liancheng Guan, ${ }^{5}$ Mengzhi Zhang, ${ }^{1}$ Huaiquan Liu, ${ }^{1}$ Haiyang Yu, \\ Qingxue Wang, ${ }^{1}$ and Changfu Yang $\mathbb{1}^{1}$ \\ ${ }^{1}$ Department of Preclinical Medicine, Guizhou University of Traditional Chinese Medicine, Guiyang, Guizhou, China \\ ${ }^{2}$ Center for Traditional Chinese Ethnic Minority Medicine, Guizhou University of Traditional Chinese Medicine, Guiyang, Guizhou, China \\ ${ }^{3}$ Center for Translational Medicine, Guizhou University of Traditional Chinese Medicine, Guiyang, Guizhou, China \\ ${ }^{4}$ Department of Pharmacy, Guizhou University of Traditional Chinese Medicine, Guiyang, Guizhou, China \\ ${ }^{5}$ Second Affiliated Hospital, Guizhou University of Traditional Chinese Medicine, Guiyang, Guizhou, China
}

Correspondence should be addressed to Changfu Yang; 435140961@qq.com

Received 25 April 2020; Accepted 21 July 2020; Published 21 August 2020

Academic Editor: Norhaizan Mohd Esa

Copyright (c) 2020 Yihui Chai et al. This is an open access article distributed under the Creative Commons Attribution License, which permits unrestricted use, distribution, and reproduction in any medium, provided the original work is properly cited.

Background. Aidi injection (ADI) is being used widely for breast cancer in China. However, the efficacy and safety of it need to be summarized. We conducted a systematic review and meta-analysis to compare ADI and non-ADI treatment for advanced breast cancer. Methods. We searched PubMed, EMBASE, CNKI, SinoMed, and CENTRAL from inception to Jan 2020 for randomized controlled trials (RCTs) with diagnosis of advanced breast cancer that compared the efficacy of ADI with non-ADI treatment. Two researchers screened the literature, extracted data, and evaluated risk of bias separately. The primary outcomes were overall response rate (ORR) and disease control rate (DCR). The secondary outcomes included the QOL, immune cells, and adverse events. Review Manager software was used for estimating risks of bias of included studies, data analysis, and plotting. The sensitivity analysis and the publication bias test were performed using the $R$ language. $I^{2}$ and chi-square tests were used to estimate heterogeneity. If $P>0.1$ or $I^{2}<40 \%$, the fixed-effect model meta-analysis was performed. A random or fixedeffect analysis was used depending on the heterogeneity testing. Weighted mean difference (WMD) or standard mean difference (SMD) was used for analysis of continuous data, and the rate ratio (RR) was calculated for the dichotomous variable, respectively. Results. We included 14 studies with 1006 patients diagnosed as advanced breast cancer in total. The pooled effect showed that ADI increased ORR in advanced BC patients as an add-on therapy with little heterogeneity $(R R=1.14,95 \%$ CI $1.03-1.27)$. DCR in BC patients could not be improved by ADI. ADI improved the KPS score in BC patients compared with chemotherapy alone $(\mathrm{MD}=3.26$, 95\% CI 1.74-4.78). There were no improvements on immune markers except CD4/CD8 and NK\%. Serum tumor markers CEA and CA153 were decreased while treated with ADI, but only one trial was involved. ADI decreased the numbers of myelosuppression in advanced BC patients, and AST, ALT, $\gamma$-GT, and CK-MB were all decreased. The sensitivity evaluation indicated that the result of the pooled effect size had good stability. Conclusion. This meta-analysis suggested that based on the existing evidence, treatment with ADI significantly changed the ORR of patients with advanced BC and improved their quality of life with few side effects. However, more randomized trials involving larger samples should be considered, and detailed mechanisms are needed to be uncovered.

\section{Introduction}

Breast cancer (BC) is one of the most usual malignant tumors among women worldwide which results in high rates of morbidity and mortality $[1,2]$. Over the past two decades, the incidence rate of $\mathrm{BC}$ has been increasing constantly $[3,4]$. Accumulating evidence showed that genes, proteins, and several pathways are involved in the occurrence and 
progression of $\mathrm{BC}$, and the precise molecular mechanisms are still unclear. To date, surgery is the first choice for earlystage BC patients, but most clinically diagnosed advanced BC patients are forced to accept chemotherapy [5], radiotherapy [6], endocrine therapy [7], or biotherapy [8]. The management of the disease is primarily to improve quality of life (QOL) and prevent disease from recurring.

Although targeted add-on therapy with monoclonal antibodies such as trastuzumab or pertuzumab has been proved to be efficacious in specific types of BC, the high costs still slowed down the widespread use in developing countries of the world. Therefore, effective and affordable adjunct therapies are needed. Aidi injection (ADI) is a compound preparation injection of Chinese herbs (Z52020236, CFDA), which is composed of the extracts from Panax ginseng C. A. Mey, Astragalus propinquus Schischkin, Acanthopanax senticosus (Rupr. Maxim.) Harms, and Mylabris phalerata Pallas [9]. According to a study on chemical constituents in the Aidi injection, 22 chemical components were detected and isolated [10]. These compounds are astragaloside, ginsenoside, eleutheroside, coniferin, etc. Previous studies showed that ADI could significantly improve the clinical response and QOL in patients with non-small cell lung cancer (NSCLC) [11] and gastric cancer. Several clinical trials also revealed that ADI could reduce the toxicity of chemotherapy in breast cancer $[12,13]$. However, the efficacy on BC has been inconclusive due to a lack of summary. Therefore, we performed a systematic review and metaanalysis. Findings from such a study may help determine whether to use ADI as an add-on therapy on BC.

\section{Methods}

2.1. Protocol and Registration. The protocol of the present review was registered in the International Platform of Registered Systematic Review and Meta-Analysis Protocols (Inplasy, https://inplasy.com/) and was reported in accordance with PRISMA [14] (Preferred Reporting Items for Systematic Reviews and Meta-Analyses). The registration number is INPLASY202040170, and the DOI number is 10.37766/inplasy2020.4.0170.

2.2. Search Strategy. We conducted an online search for trials from inception up to Jan 2020, in PubMed (https:// www.ncbi.nlm.nih.gov/pubmed), EMBASE (http://www. embase.com), CNKI (http://www.cnki.net/), SinoMed (http://www.sinomed.ac.cn/), and the Cochrane Central Register of Controlled Trials (CENTRAL) (http:// onlinelibrary.wiley.com/cochranelibrary/) with the search terms "(Breast Neoplasms [MH] OR breast neoplasm* [TIAB] OR breast carcinoma*[TIAB] OR breast tumor* [TIAB] OR breast tumor* [TIAB] OR breast cancer* [TIAB]) AND (Aidi injection[TIAB])," following the demonstration of Cochrane handbook (ZM and LH). In addition, we performed handsearches of the references of all identified articles and relevant reviews (GJ).

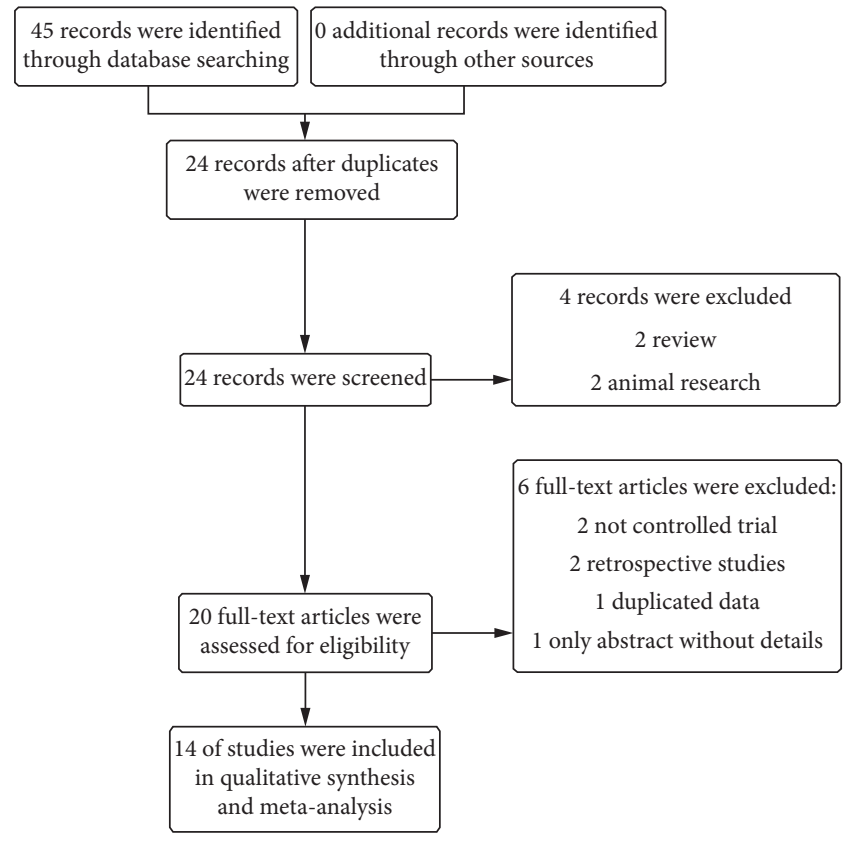

FIGURE 1: Search and selection of clinical trials assessing the efficacy and safety of ADI on advanced BC.

2.3. Study Selection. Eligible clinical trials were defined based on the following criteria: (1) randomized controlled trials of advanced breast cancer (parallel groups or crossover design); (2) age >18 years; (3) intervention with Aidi injection as an add-on therapy compared with conventional chemotherapy; (4) reported ORR and adverse events or at least one additional outcome.

Exclusion criteria: (1) animal or cell research; (2) observational studies; (3) reviews, letter to the editor, or case reports; (4) duplicates.

Two authors, respectively, reviewed the titles and abstracts (LW and CY). If there were discrepancies between the present reviewers, another author (QZ) was consulted to reach a consensus as the third investigator.

2.4. Data Collection Process. We extracted data from each selected study, including the name of the first author, publication year, geographical location, study design, cases, participants, doses, outcomes, and statistical methods. We followed the recommendations for reporting by the Preferred Reporting Items for Systematic Reviews and MetaAnalyses guidelines [14] (PRISMA). The quality of individual records was assessed according to the Cochrane handbook.

2.5. Outcomes. The primary endpoint was the overall response rate [15] (complete remission + partial remission, ORR) and the disease control rate (complete remission + partial remission + stable disease, DCR). Secondary outcomes included the QOL, immune cells, and adverse events. 
TABLE 1: Characteristics of included trials.

\begin{tabular}{|c|c|c|c|c|c|c|c|c|}
\hline Trials & Design & $\begin{array}{c}\text { No. of } \\
\text { cases T/C }\end{array}$ & Age $\mathrm{T} / \mathrm{C}$ & KPS T/C & TNM & Treatment & Control & Outcomes \\
\hline Yumeng [16] & RCT & $24 / 26$ & $\begin{array}{c}55.08 \pm 10.32 / \\
54.12 \pm 10.75\end{array}$ & $\begin{array}{c}86.25 \pm 5.76 / \\
87.50 \pm 5.52\end{array}$ & I-IV & $\begin{array}{c}\text { Aidi } 100 \mathrm{ml} / \mathrm{d} / 1-7 \\
\mathrm{q} 21 \mathrm{~d}+\mathrm{CEF} \text { or } \\
\text { CAF }\end{array}$ & $\begin{array}{l}\text { CEF or } \\
\text { CAF }\end{array}$ & $\begin{array}{c}\text { SAS, SDS, } \\
\text { QLQC30, ORR, } \\
\text { AEs }\end{array}$ \\
\hline Weiming [13] & RCT & $39 / 40$ & $\begin{array}{l}46.73 \pm 14.2 / \\
45.98 \pm 15.78\end{array}$ & $>50 \#$ & III-IV & $\begin{array}{c}\text { Aidi } 100 \mathrm{ml} / \mathrm{d} / 1-8 \\
\text { q } 21 \mathrm{~d}+\mathrm{CEF}\end{array}$ & $\mathrm{CEF}$ & $\begin{array}{c}\text { ICs, QoL, BMs, } \\
\text { AEs }\end{array}$ \\
\hline Yonghong [12] & RCT & $64 / 64$ & $46.7 \pm 20.3$ & \# & I-IV & $\begin{aligned} \text { Aidi } & 100 \mathrm{ml} / \mathrm{d} / 1-8 \\
\text { q } & 21 \mathrm{~d}+\mathrm{CEF}\end{aligned}$ & CEF & ICs, AEs \\
\hline Liwang et al. [17] & RCT & $78 / 62$ & $\begin{array}{c}52.5(24 \sim 76) / 51.2 \\
(20 \sim 70)^{*}\end{array}$ & $\#$ & I-IV & $\begin{array}{c}\text { Aidi } 100 \mathrm{ml} / \mathrm{d} / \\
1-14+\mathrm{CEF} \text { or } \\
\mathrm{CAF}\end{array}$ & $\begin{array}{l}\text { CEF or } \\
\text { CAF }\end{array}$ & ICs \\
\hline Mei and Li [18] & RCT & $23 / 23$ & $52(36 \sim 64)^{*}$ & $\#$ & I-III & $\begin{array}{c}\text { Aidi } 100 \mathrm{ml} / \mathrm{d} / 1-7 \\
\text { q } 21 \mathrm{~d}+\mathrm{CEF}\end{array}$ & $\mathrm{CEF}$ & VEGF \\
\hline Sandi et al. [19] & RCT & $26 / 22$ & $\begin{array}{c}42.27 \pm 6.32 / \\
42.23 \pm 6.7\end{array}$ & $\begin{array}{c}84.23 \pm 5.78 / \\
84.55 \pm 5.96\end{array}$ & IIA-IIIC & $\begin{array}{c}\text { Aidi } 60 \mathrm{ml} / \mathrm{d} / 1-4 \mathrm{q} \\
14 \mathrm{~d}+\mathrm{TC}-\mathrm{P}\end{array}$ & TC-P & ORR, QoL, AEs \\
\hline Chuanhui et al. [20] & RCT & $24 / 28$ & $\begin{array}{l}57.21 \pm 3.52 / \\
55.66 \pm 3.43\end{array}$ & \# & IIB-IIIB & $\begin{array}{c}\text { Aidi } 80 \mathrm{ml} / \mathrm{d} / 1-15 \\
\text { q } 21 \mathrm{~d}+\text { TAC }\end{array}$ & TAC & ORR, ICs \\
\hline Zhuorong et al. [21] & RCT & $30 / 26$ & $\begin{array}{c}42.47 \pm 7.85 / \\
42.54 \pm 8.10\end{array}$ & $\begin{array}{l}83.67 \pm \\
84.62 \pm\end{array}$ & II-III & $\begin{array}{c}\text { Aidi } 60 \mathrm{ml} / \mathrm{d} / 1-4 \mathrm{q} \\
14 \mathrm{~d}+\mathrm{AC}-\mathrm{T}\end{array}$ & $\mathrm{AC}-\mathrm{T}$ & ORR, QoL, AEs \\
\hline $\begin{array}{l}\text { Xiangguo and } \\
\text { Lin [22] }\end{array}$ & RCT & $28 / 20$ & $\begin{array}{l}36.2 \pm \\
37.5\end{array}$ & $\begin{array}{c}72.87 \pm 4.69 / \\
71.89 \pm 5.03\end{array}$ & I-IIIA & $\begin{array}{l}\text { Aidi } 100 \mathrm{ml} / \mathrm{d} / \\
1-10 \mathrm{q} 21 \mathrm{~d}+\mathrm{CTF}\end{array}$ & CTF & ORR, QoL, AEs \\
\hline $\begin{array}{l}\text { Ling and } \\
\text { Xiaoge [23] }\end{array}$ & RCT & $44 / 44$ & 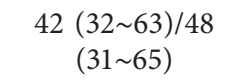 & $\#$ & I-IV & $\begin{array}{l}\text { Aidi } 100 \mathrm{ml} / \mathrm{d} / \\
1-15 \mathrm{q} 28 \mathrm{~d}+\mathrm{NP}\end{array}$ & NP & $\begin{array}{c}\text { ORR, DCR, } \\
\text { TTP, AEs }\end{array}$ \\
\hline $\begin{array}{l}\text { Xiangqiand } \\
\text { Shaobo [24] }\end{array}$ & RCT & $32 / 20$ & $\begin{array}{c}46.2 \pm 2.6 / \\
44.5 \pm 3.2\end{array}$ & $\begin{array}{c}70.78 \pm 4.40 / \\
71.19 \pm 4.53\end{array}$ & I-IIIA & $\begin{array}{l}\text { Aidi } 100 \mathrm{ml} / \mathrm{d} / \\
1-10 \mathrm{q} 21 \mathrm{~d}+\mathrm{CEF}\end{array}$ & $\mathrm{CEF}$ & ORR, QoL, AEs \\
\hline Wenjuan [25] & RCT & $30 / 30$ & $48.4 / 47.6$ & $\#$ & III-IV & $\begin{array}{l}\text { Aidi } 100 \mathrm{ml} / \mathrm{d} / \\
1-10 \mathrm{q} 21 \mathrm{~d}+\mathrm{CAF}\end{array}$ & CAF & ORR, ICs, QoL \\
\hline Zhenzhen [26] & RCT & $50 / 50$ & $45^{*}$ & $\#$ & II-III & $\begin{array}{l}\text { Aidi } 100 \mathrm{ml} / \mathrm{d} / \\
1-14 \text { q } 21 \mathrm{~d}+\mathrm{CAF}\end{array}$ & CAF & $\begin{array}{c}\text { ORR, ICs, QoL, } \\
\text { AEs }\end{array}$ \\
\hline Ling [27] & RCT & $31 / 28$ & $\begin{array}{c}54.2(32 \sim 69) / 53.5 \\
(31 \sim 70)\end{array}$ & \# & II-IV & $\begin{array}{c}\text { Aidi } 50 \mathrm{ml} / \mathrm{d} / 1-15 \\
\text { q } 21 \mathrm{~d}+\mathrm{NT}\end{array}$ & NT & $\begin{array}{c}\text { ORR, ICs, QoL, } \\
\text { AEs }\end{array}$ \\
\hline
\end{tabular}

BC: breast cancer; T: treatment; C: control; ORR: overall response rate; DCR: disease control rate; TTP: time to progression; AE: adverse events; QoL: quality of life; BM: blood marker; IC: immune cell; SAS: Self-Rating Anxiety Scale; SDS: Self-Rating Depression Scale; QLQC30: Quality Of Life Questionnaire Core 30; CF: cardiac function; ECG: electrocardiogram; CK: creatinine kinase; CEF: cytoxan, epirubicin, and 5-fluorouracil; CAF: cytoxan, adriamycin, and 5-fluorouracil; TCP: theprubicin, cytoxan, and paclitaxel; TAC: theprubicin, adriamycin, and cytoxan; AC-T: adriamycin, cytoxan, and theprubicin; CTF: cytoxan, theprubicin, and 5-fluorouracil; NT: navelbine and theprubicin. ${ }^{*}$ Data were expressed as medium and interquartile range (IQR). \#Details not reported.

2.6. Statistical Analysis. Review Manager software (version 5.3; Cochrane Collaboration, Oxford, UK) was used for estimating risks of bias of included studies, data analysis, and plotting. The sensitivity analysis and the publication bias test were performed using the $R$ language. $I^{2}$ and chi-square tests were used to estimate heterogeneity. If $P>0.1$ or $I^{2}<40 \%$, the fixed-effect model meta-analysis was performed. When there was a high degree of heterogeneity, a random-effect analysis was used. For each group, the Aidi injection group was compared to placebo or other active chemotherapy. Weighted mean difference (WMD) or standard mean difference (SMD) was used for analysis of continuous data, and the rate ratio (RR) was calculated for the dichotomous variable, respectively.

\section{Results}

3.1. Study Description and Risk of Bias. By using the search strategy mentioned above, a total of 24 trials were identified after duplicated records were removed. After screening the title and the abstracts, we retrieved the full texts of 20 records, of which 14 were ultimately included in our analysis involving 1006 participants totally. The details of the exclusions are shown in Figure 1. In total, 14 trials were included in the present study, and the characteristics of the trials are shown in Table 1. Most of the included trials showed relatively low to medium quality. The Cochrane handbook [28] was used to evaluate the risk of bias for RCTs (Figure 2). The treatments of the 14 included articles were ADI plus chemotherapy.

3.2. Primary Outcomes. Ten trials reported ORR and DCR as the main outcome. The pooled effect showed that ADI increased ORR in BC patients as an add-on therapy with little heterogeneity $\left(R R=1.14,95 \%\right.$ CI $1.03-1.27 ; \mathrm{chi}^{2}=5.71$, $P=0.77 ; I^{2}=0 \%$; Figure 3$)$. DCR in BC patients could not be improved by ADI as an add-on therapy $(R R=1.02,95 \% \mathrm{CI}$ $0.97-1.07 ; \mathrm{chi}^{2}=6.55, P=0.6 ; I^{2}=0 \%$; Figure 4 ).

3.3. Secondary Outcomes. ADI plus chemotherapy improved the KPS score in BC patients compared with chemotherapy alone $\left(\mathrm{MD}=3.26,95 \%\right.$ CI 1.74-4.78; $\mathrm{chi}^{2}=0.4, P=0.94$; $I^{2}=0 \%$; Figure 5). There were no improvements on CD3\%, 


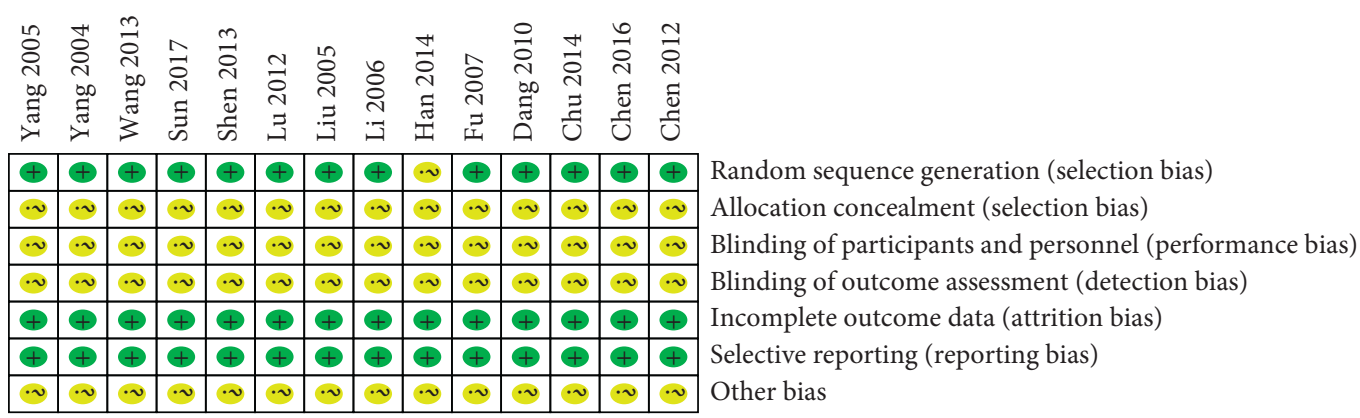

Figure 2: Risk of bias of included studies.

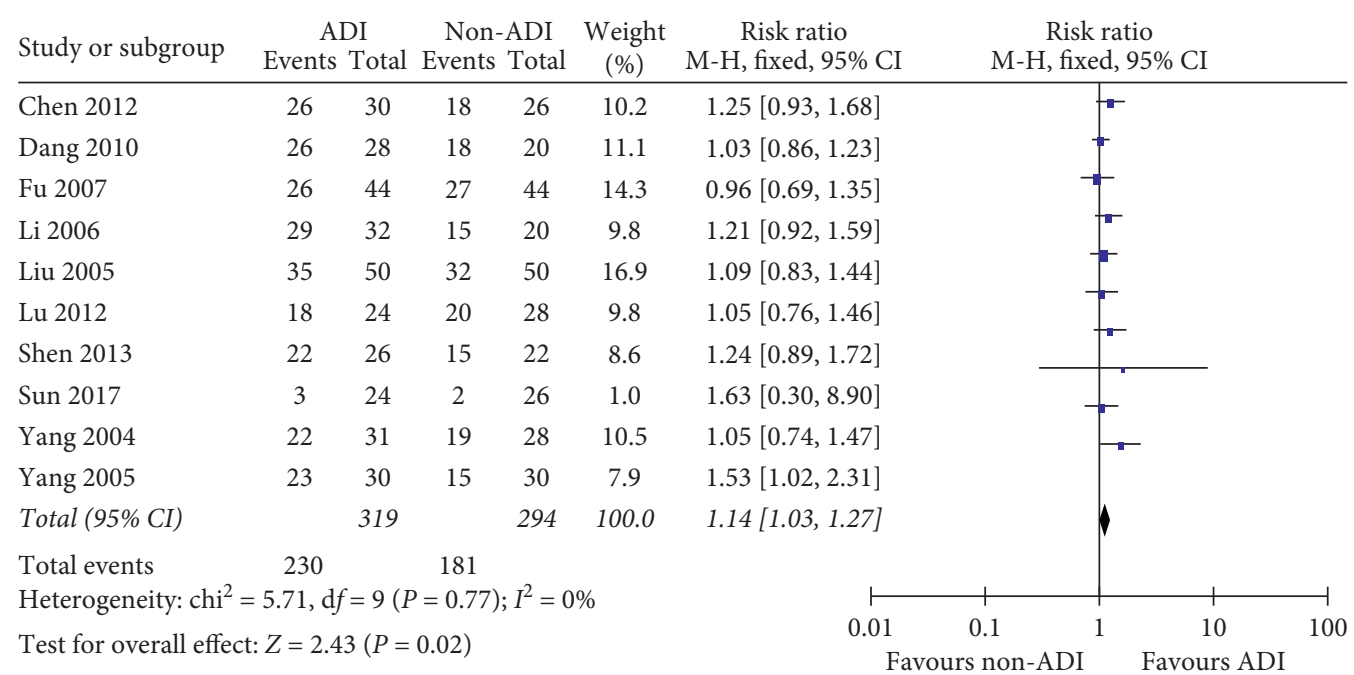

FIGURE 3: ADI increased ORR in advanced BC patients as an add-on therapy $\left(R R=1.14,95 \%\right.$ CI $\left.1.03-1.27 ; \operatorname{chi}^{2}=5.71, P=0.77 ; I^{2}=0 \%\right)$.

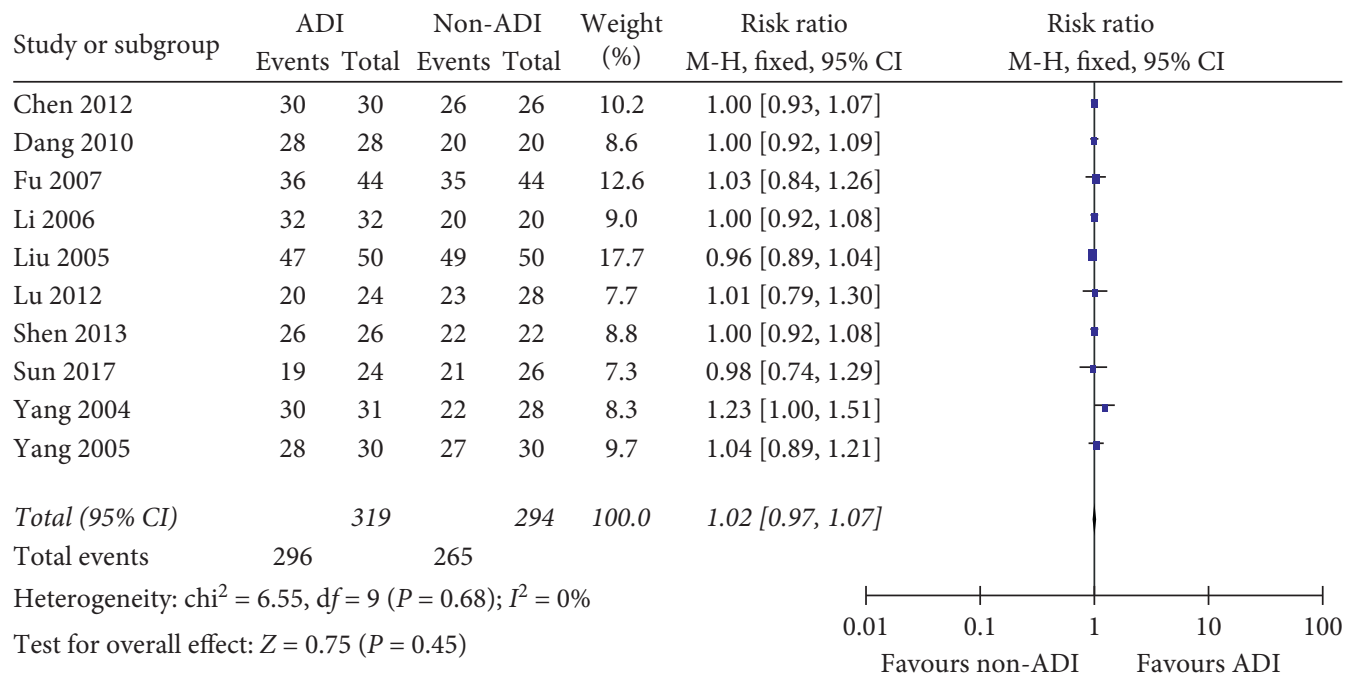

FIgURE 4: ADI did not improve DCR in advanced BC patients as an add-on therapy $\left(R R=1.02,95 \%\right.$ CI $\left.0.97-1.07 ; \operatorname{chi}^{2}=6.55, P=0.68 ; I^{2}=0 \%\right)$.

$\mathrm{CD} 4 \%$, and $\mathrm{CD} 8 \%$. The CD4/CD8 ratio was higher while treated with ADI with a high heterogeneity $(\mathrm{MD}=0.32,95 \%$ CI $\left.0.07-0.58 ; \mathrm{chi}^{2}=48.88, P \leq 0.01 ; I^{2}=88 \%\right)$. NK\% data showed the same trend with CD4/CD8. Serum tumor markers CEA and CA153 were decreased while treated with ADI, but only one trial was involved (Table 2).
3.4. Adverse Events. ADI decreased the numbers of myelosuppression in advanced BC patients as an add-on therapy $\left(R R=0.69,95 \%\right.$ CI $0.52-0.92 ; \mathrm{chi}^{2}=17.95, P=0.003$; $I^{2}=72 \%$; Figure 6). AST, ALT, $\gamma$-GT, and CK-MB were all decreased by ADI treatment. No other side effects were recorded during the studies. Details are shown in Table 3. 


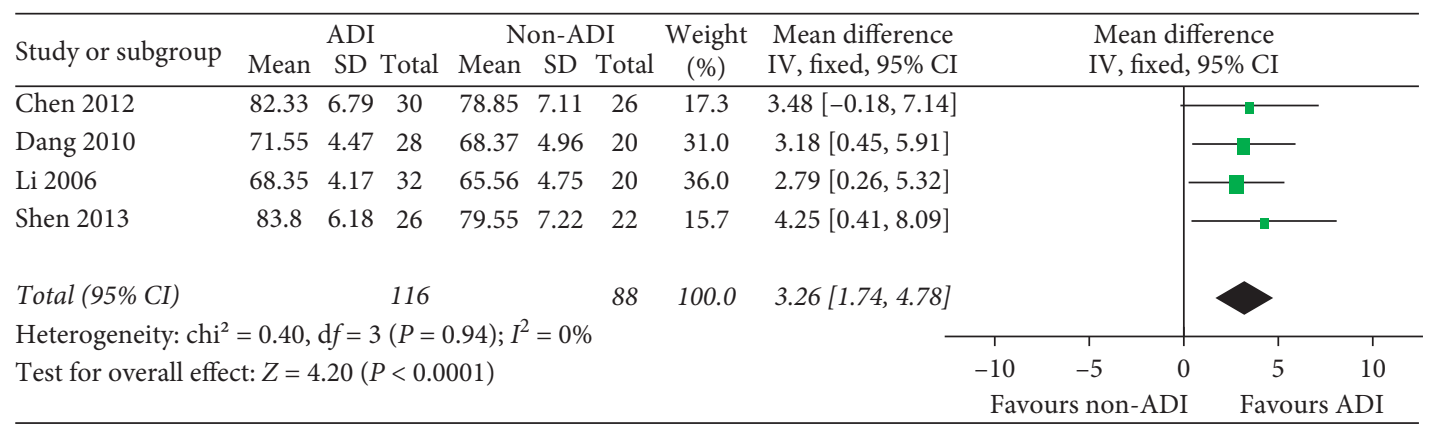

FIGURE 5: ADI improved the KPS score in advanced BC patients as an add-on therapy $\left(\mathrm{MD}=3.26,95 \% \mathrm{CI} 1.74-4.78 ; \mathrm{chi}^{2}=0.4, P=0.94\right.$; $\left.I^{2}=0 \%\right)$.

TABLE 2: Secondary outcomes.

\begin{tabular}{lccccc}
\hline \multirow{2}{*}{ Outcomes } & \multirow{2}{*}{ No. of trials } & \multicolumn{2}{c}{ Heterogeneity } & Effect size with 95\% CI & Z with $P$ value \\
\hline Immune cells & & Chi-squared & $I$-squared $(\%)$ & \\
CD3\% & $7^{12,13,18,21,26-28}$ & $514.87(P<0.00001)$ & 99 & $3.71(-3.85 \sim 11.27)$ & $0.96(P=0.34)$ \\
CD4\% & $7^{12,13,18,21,26-28}$ & $1302.78(P<0.0001)$ & 100 & $6.67(-2.71 \sim 16.06)$ & $1.39(P=0.16)$ \\
CD8\% & $7^{12,13,18,21,26-28}$ & $747.57(P<0.00001)$ & 99 & $-0.97(-7.54 \sim 5.6)$ & $0.29(P=0.77)$ \\
CD4/CD8 & $7^{12,13,18,21,26-28}$ & $48.88(P<0.00001)$ & 88 & $0.32(0.07 \sim 0.58)$ & $2.5(P=0.01$ \\
NK\% & $4^{12,13,18,26}$ & $29.39(P<0.00001)$ & 90 & $5.54(4.60 \sim 6.47)$ & $11.64(P<0.001)$ \\
\hline Tumor markers & & - & - & $-2.39(-3.99 \sim-0.79)$ & $2.93(P=0.003)$ \\
CEA & $1^{13}$ & - & - & $-3.06(-5.17 \sim-0.95)$ & $2.85(P=0.004)$ \\
CA153 & $1^{13}$ & - & &
\end{tabular}

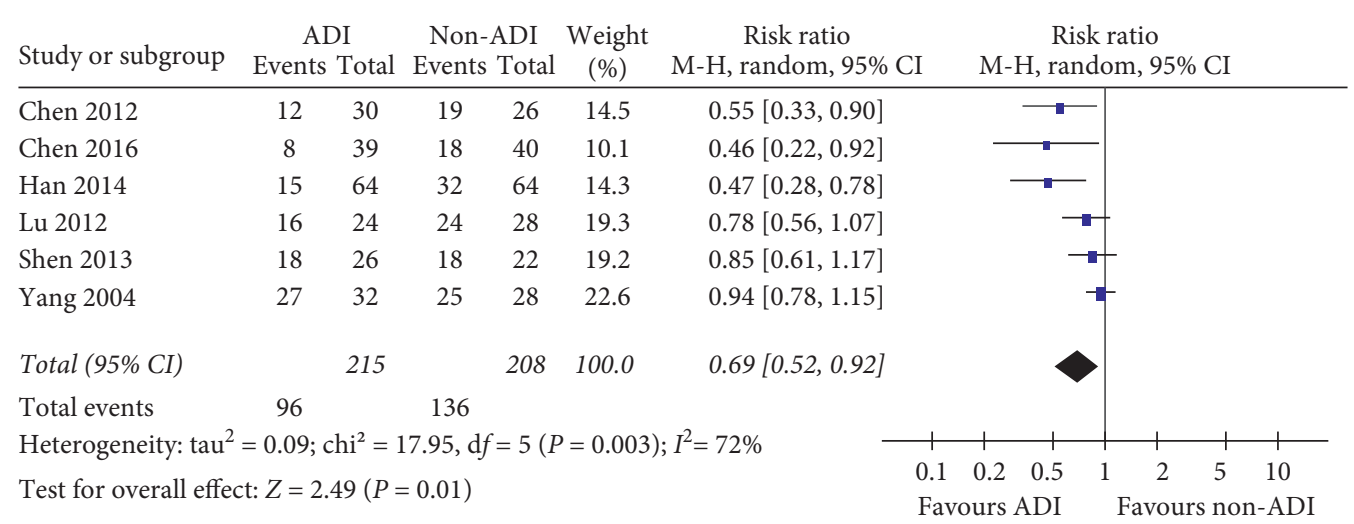

FIGURE 6: ADI decreased the numbers of myelosuppression in BC patients as an add-on therapy $\left(R R=0.69 ; 95 \%\right.$ CI $0.52-0.92 ; I^{2}=72 \%$; $P=0.003$ ).

TABLe 3: Safety of ADI.

\begin{tabular}{lccccc}
\hline \multirow{2}{*}{ Outcomes } & \multirow{2}{*}{ No. of trials } & \multicolumn{2}{c}{ Heterogeneity } & Effect size with 95\% CI & $Z$ with $P$ value \\
\hline Hepatic function & & & & & \\
AST & $3^{22,23,25}$ & $112.69(P<0.00001)$ & 98 & $-31.21(-47.06 \sim-15.36)$ & $3.86(P=0.001)$ \\
ALT & $3^{22,23,25}$ & $4.86(P=0.09)$ & 59 & $-4.04(-5.57 \sim-2.51)$ & $5.16(P<0.0001)$ \\
$\gamma$-GT & $2^{23,25}$ & $0.08(P=0.78)$ & 0.0 & $-24.59(-27.78 \sim-21.40)$ & $15.1(P<0.0001)$ \\
\hline Cardiac function & & & & & \\
CK-MB & $2^{22,23}$ & $0.64(P=0.42)$ & 0.0 & $-4.04(-5.91 \sim-2.17)$ & $4.23(P<0.0001)$ \\
\hline
\end{tabular}


3.5. Publication Bias. No obvious publication bias was found through the funnel plot (ORR) (Figure 7). Egger's test showed the result of the linear regression test of the funnel plot asymmetry: $t=1.4319, d f=8$, and $P$ value $=0.1901$. The result indicated that there was no publication bias.

3.6. Sensitivity Analysis. The sensitivity was evaluated through excluding the poor and overestimated studies about the main outcome ORR. The analysis indicated that the result of the pooled effect size had good stability (Figure 8).

\section{Discussion}

$\mathrm{BC}$ is commonly discovered among women worldwide of which the incidence rate has been increasing constantly. Advanced breast cancer patients do not have many choices but to accept chemotherapy. During a long-term clinical practice, traditional Chinese medicines have played important roles in treating some types of tumors. However, the molecular mechanisms are poorly discovered. ADI is a compound injection of Chinese herbs which is widely used in treating malignant tumors including breast cancer.

The present meta-analysis suggested that based on the existed evidence, treatment with ADI significantly changed the ORR of patients with advanced BC but did not obviously increase the DCR. There were also improvements on quality of life, and an increase in the KPS score was observed. To some extent, the immune system was improved because the CD4/CD8 ratio and NK cells were higher while treated with ADI. However, CD3, CD4, and CD8 did not change because of one study [26] which induced high heterogeneity and showed totally reversed effect to other studies. The participant's age, intervention, and duration were not significantly different from others'. ADI seemed to be safe for patients. ADI decreased the numbers of myelosuppression, AST, ALT, $\gamma$-GT, and CK-MB in BC patients as an add-on therapy. No obvious publication bias was found through the funnel plot (ORR).

The mechanism of ADI on BC was suggested that ADI significantly inhibited the proliferation of MCF-7 cells in a dose-dependent manner [29] and the miRNA might serve as potentially therapeutic targets. The modulation of miRNA expression is an important mechanism of ADI inhibiting breast cancer cell growth. Another experiment reported that ADI could inhibit proliferation, promote apoptosis and necrosis of tumor cells, and significantly reduce the cell diameter [30]. But, the research is limited, so more studies should better be involved in and discover the underlying mechanisms.

Several limitations of this meta-analysis should be mentioned. First, the quality of included trials was relatively low, some of which did not report the details of blinding and allocation concealment. This might induce bias of the results. Little research studies provided survival data. Previous studies showed good effect of ADI on patients with BC which may be a potential drug as an adjunct therapy. However, additional high-quality RCTs and larger sample sizes may lead to more reliable results. Second, the records of

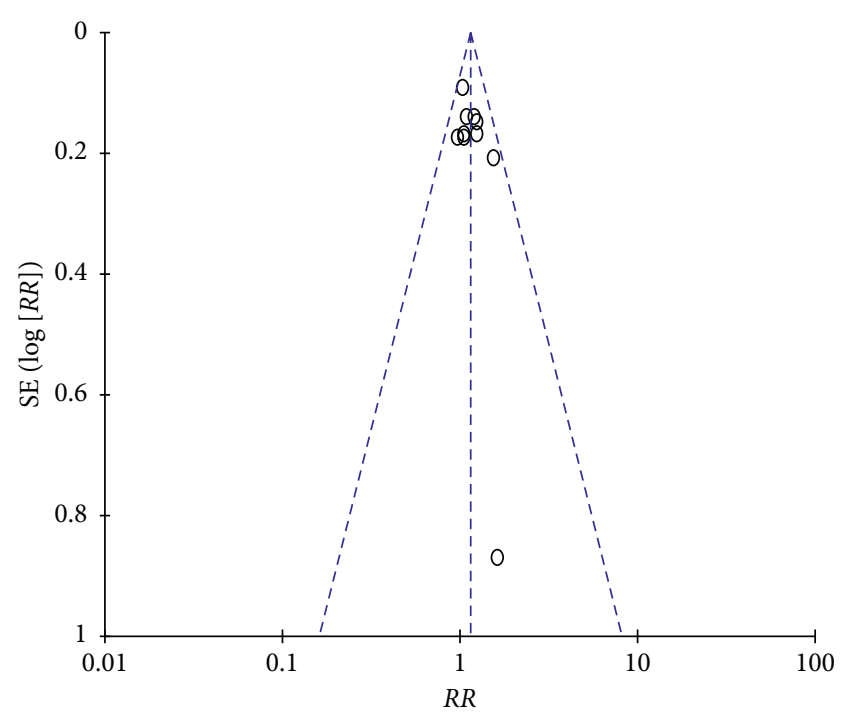

Figure 7: Publication bias. There was no publication bias in the included studies.

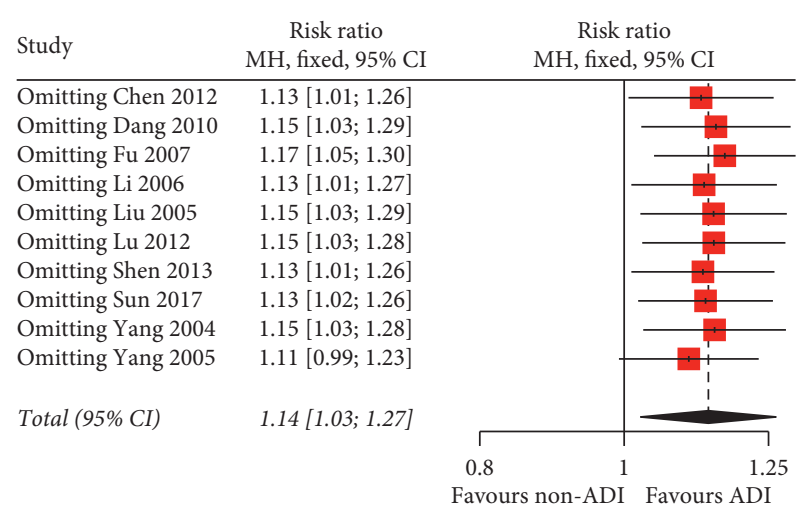

FIGURE 8: Sensitivity analysis showing that the result had good stability.

survival terms were seldomly reported, and we were not able to calculate the overall survival of specific year. Furthermore, the therapeutic duration and designs were not identical, which may lead to heterogeneity. Subgroup analysis was not performed because included articles were limited and difficult to be grouped.

\section{Conclusion}

In summary, this meta-analysis suggested that based on the existing evidence, treatment with ADI significantly changed the ORR of patients with advanced BC and improved their quality of life with few side effects. More randomized trials involving larger samples should be considered, and detailed mechanisms are needed to be uncovered.

\section{Conflicts of Interest}

The authors declare that there are no conflicts of interest regarding the publication of this paper. 


\section{Authors' Contributions}

Yihui Chai, Yunzhi Chen, and Wen Li contributed equally to this work.

\section{Acknowledgments}

This work was supported by the National Natural Science Foundation of China (nos. 81760841, 81874376, and 81660762), the Research Projects on Science and Technology of Science and Technology Program in Guizhou Province (Qiankehe Platform Talents [2017]5735-12), and the Project of Education Department of Guizhou Province (K[2017]041 and KY[2017]172).

\section{References}

[1] A. Kolak, M. Kamińska, K. Sygit et al., "Primary and secondary prevention of breast cancer," Annals of Agricultural and Environmental Medicine, vol. 24, no. 4, pp. 549-553, 2017.

[2] C. G. Yedjou, P. B. Tchounwou, M. Payton et al., "Assessing the racial and ethnic disparities in breast cancer mortality in the United States," International Journal of Environmental Research and Public Health, vol. 14, no. 5, 2017.

[3] M. Wang, C. Zhang, Y. Song et al., "Mechanism of immune evasion in breast cancer," OncoTargets and Therapy, vol. 10, pp. 1561-1573, 2017.

[4] L. Xing, Q. He, Y.-Y. Wang, H.-Y. Li, and G.-S. Ren, “Advances in the surgical treatment of breast cancer," Chinese Clinical Oncology, vol. 5, no. 3, p. 34, 2016.

[5] D. J. Butters, D. Ghersi, N. Wilcken, S. J. Kirk, and P. T. Mallon, "Addition of drug/s to a chemotherapy regimen for metastatic breast cancer," Cochrane Database of Systematic Reviews, vol. 11, 2010.

[6] P. J. Hoskin, K. Hopkins, V. Misra et al., "Effect of singlefraction vs multifraction radiotherapy on ambulatory status among patients with spinal canal compression from metastatic cancer," Journal of the American Medical Association, vol. 322, no. 21, pp. 2084-2094, 2019.

[7] O. Najim, S. Seghers, L. Sergoynne et al., "The association between type of endocrine therapy and development of estrogen receptor-1 mutation(s) in patients with hormonesensitive advanced breast cancer: a systematic review and meta-analysis of randomized and non-randomized trials," Biochimica et Biophysica Acta (BBA)-Reviews on Cancer, vol. 1872, no. 2, Article ID 188315, 2019.

[8] K. Ishii, N. Morii, and H. Yamashiro, "Pertuzumab in the treatment of HER2-positive breast cancer: an evidence-based review of its safety, efficacy, and place in therapy," Core Evidence, vol. 14, pp. 51-70, 2019.

[9] Z. Xiao, C. Wang, M. Zhou et al., "Clinical efficacy and safety of Aidi injection plus paclitaxel-based chemotherapy for advanced non-small cell lung cancer: a meta-analysis of 31 randomized controlled trials following the PRISMA guidelines," Journal of Ethnopharmacology, vol. 228, pp. 110-122, 2019.

[10] Z. Miaomiao, L. Yanli, C. Zhong, L. Xiaoran, X. Qiongming, and Y. Shilin, "Studies on chemical constituents from Aidi injection," Chinese Traditional and Herbal Drugs, vol. 43, no. 8, pp. 1462-1470, 2012.

[11] Z. Xiao, C. Wang, R. Zhou et al., "Can Aidi injection improve overall survival in patients with non-small cell lung cancer? A systematic review and meta-analysis of 25 randomized controlled trials," Complementary Therapies in Medicine, vol. 37, pp. 50-60, 2018.

[12] H. Yonghong, J. Xueqing, Y. Ye, W. Zhiyong, and J. Ming, "Clinical observation of Aidi injection combined with CEF regimen chemotherapy in treatment of postoperative breast cancer," Hubei Journal of Traditional Chinese Medicine, vol. 36, no. 05, pp. 7-8, 2014.

[13] C. Weiming, "Clinical observation of Aidi injection combined with CEF chemotherapy in the treatment of breast cancer after operation," Chinese Journal of Modern Drug Application, vol. 10, no. 15, pp. 185-187, 2016.

[14] D. Moher, A. Liberati, J. Tetzlaff, and D. G. Altman, "Preferred reporting items for systematic reviews and metaanalyses: the PRISMA statement," British Medical Journal, vol. 339, 2009.

[15] V. R. Preedy and R. R. Watson, "Overall response rate," in Handbook of Disease Burdens and Quality of Life Measures, Springer, New York, NY, USA, 2010.

[16] S. Yumeng, Clinical Observation on Improving Mood and Quality of Life of Breast Cancer Patients with Aidi Injection [ \pm ], Liaoning University of Chinese Medicine, Shenyang, China, 2017.

[17] C. Liwang, W. Zongyan, Z. Liangang, P. Yuzhu, and G. Weiliang, "Effect of Aidi injection on cellular immunity in breast cancer patients," China Medical Engineering, vol. 21, no. 12, pp. 36-37, 2013.

[18] W. Mei and W. Li, "Effect of Aidi injection on serum VEGF level in breast cancer patients," Chinese Journal of Clinical Research, vol. 26, no. 11, pp. 1151-1158, 2013.

[19] S. Sandi, C. Zhuorong, X. Gaofang, L. Zhihui, and H. Zhan, "Clinical observation of dose-intensive TC-P combined with Aidi injection in the treatment of triple negative breast cancer," Guide of China Medicine, vol. 11, no. 8, pp. 84-85, 2013.

[20] L. Chuanhui, H. Ming, Y. Jun, and X. Lin, "Clinical observation of Aidi injection combined with TAC regimen in preoperative neoadjuvant chemotherapy for breast cancer," Traditional Chinese Medicine Journal, vol. 11, no. 3, pp. 50-52, 2012.

[21] C. Zhuorong, S. Sandi, H. Zhan, L. Ruiwen, and L. Yanming, "Clinical observation of dose-intensive AC-T regimen combined with Aidi injection in the treatment of triple negative breast cancer," Chin Journal of Surgery Oncology, vol. 4, no. 2, pp. 85-87, 2012.

[22] D. Xiangguo and W. Lin, "Evaluation of efficacy on breast cancer treated by Aidi injection plus CTF Program of neoadjuvant chemotherapy and the impacts on serum sFas," World Journal of Integrated Traditional and Western Medicine, no. 1, pp. 54-56, 2010.

[23] F. Ling and K. Xiaoge, "Aidi injection combined with chemotherapy for 44 cases of advanced breast cancer," Jouranl of Practical Traditional Chinese Medicine, vol. 23, no. 8 , p. $517,2007$.

[24] L. Xiangqi and G. Shaobo, "Efficacy analysis of Aidi injection combined with CEF chemotherapy for breast cancer," Pharmacology and Clinics of Chinese Materia Medica, vol. 22, no. C1, pp. 176-177, 2006.

[25] Y. Wenjuan, "Aidi injection combined with CAF chemotherapy for 30 cases of advanced breast cance," Jiangxi Journal of Traditional Chinese Medicine, vol. 36, no. 7, pp. 46-47, 2005.

[26] L. Zhenzhen, L. Hui, L. Lianfang, and C. Shude, "Clinical observation of Aidi injection combined with chemotherapy in the treatment of locally advanced breast cancer," Shandong Medical Journal, vol. 45, no. 19, p. 62, 2005. 
[27] Y. Ling, "Clinical observation of Aidi injection combined with chemotherapy in the treatment of advanced breast cancer," Chinese Journal of Integrated Traditional and Western Medicine, vol. 24, no. 8, pp. 755-756, 2004.

[28] J. P. Higgins and S. Green, Cochrane Handbook for Systematic Reviews of Interventions, Cochrane Book Series, London, UK, 2008.

[29] H. Zhang, Q.-M. Zhou, Y.-Y. Lu, J. Du, and S.-B. Su, "Aidi injection () alters the expression profiles of MicroRNAs in human breast cancer cells," Journal of Traditional Chinese Medicine, vol. 31, no. 1, pp. 10-16, 2011.

[30] T. Jing and W. Guojun, "Antitumor effect of Aidi injection on ErbB2 positive breast cancer cells cultured in vitro," Pharmaceutical Journal of Chinese People's Liberation Army, vol. 34, no. 4, pp. 333-340, 2018. 